Available online @ https://jiem.jnnce.ac.in https:www.doi.org/10.37314/JJEM.2021.050212 Indexed in International Scientific Indexing (ISI) Impact factor: 1.395 for 2021-22 Published on: 31 January 2022

\title{
Darcy-Forchheimer Convective Flow of Casson nanofluid in the Microchannel: Buongiorno Model
}

\author{
P. Venkatesh ${ }^{2 *}$, A. Felicita ${ }^{1}$, B. J. Gireesha ${ }^{1}$ and B. Nagaraja ${ }^{3}$ \\ 1. Department of Studies and Research in Mathematics,Kuvempu University, Shankaraghatta, \\ Shivamogga, Karnataka, India.
}

2. Department of Mathematics, Sahyadri Science College, Shivamogga, Karnataka, India.

3. Department of Mathematics, Presidency University, Bangalore, Karnataka, India.

venspv@gmail.com,felcitaalmeida@gmail.com,bjgireesu@gmail.com, nagaraj.kalapanahalli@gmail.com

\begin{abstract}
The current examination delves into the Casson nanofluid flow in the vertical microchannel. The model employed in this investigation is Buongiorno model which emphasizes the light on Brownian motion and thermophoresis effects occurring during the fluid flow. The microchannel walls are constructed in such a way so that they facilitate the suction and injection of the fluid simultaneously. Porous media is incorporated using Darcy-Forchheimer model. Involving these effects governing equations are modeled which is solved using Runge-Kutta Fehlberg $4^{\text {th }}-5^{\text {th }}$ order method. Entropy generation and Bejan number are also obtained for the concerned flow to record irreversibilities in the microchannel. The findings of this examination depict that rise in Casson parameter augments the flow velocity but causes depletion in Bejan number. On packing the microchannel with high porosity, the velocity magnifies. Both Brownian motion and thermophoresis parameter magnifies the temperature.
\end{abstract}

Keywords: Casson fluid, Buongiorno model, Darcy Forchheimer model, convective boundary, microchannel

\section{Introduction}

Darcy Forchheimer flow finds its applications in manufacturing processes in the industries which involve pollution of ground water, generation of crude oil and in propulsion devices of missiles, satellites and other space vehicles. Darcy law is formulated by making use of homogenization but while focussing on relation between Darcy's velocity and pressure gradient there exists the non-linearity and this term appears to be quadratic. Inertial impacts in a porous medium when Reynolds number is kept moderate are responsible for this non-linear term. The very existence of this term was noted by Forchheimer [1]. Darcy Forchheimer equation are used to determine high velocity flow in the porous media.

Girault and Wheeler [2] discretized
Darcy-Forchheimer model numerically. They have solved the non-linearity in the equation by using an alternating-directions algorithm. Knabner and Roberts [3] mathematical analysed a discrete fracture model in which they have studied the flow in the fracture implementing Darcy-Forchheimer flow. They have attained the existence and uniqueness of the solution for the described flow. Saif et al [4] examined the flow utilising this model over a bent stretching surface. Nayak et al [5] conducted an optimization of entropy on Darcy-Forchheimer SWCNT/MWCNT flow. Thermal field were noted to magnify for higher porosity and temperature ratios in their study.

Casson fluid model is the model which best describes the flow of blood in the small and narrow blood vessels. This model finds its 
purpose in constructing the model for removing the waste and impurities from the bloodstream before reaching the human body. This has drawn the researcher to take up the work in this model. Casson [6] analysed the Casson fluid flow over a vertically placed surface in the presence of chemical reaction and magnetic field. Mustafa et al. [7] also worked in the same direction, but over a moving plate with a parallel free stream. They have shown that the reducing Nusselt number is an elevating function of stretching ratio. Mukhopadhyay [8] came up with the Casson fluid flow and heat transfer upon a nonlinearly stretched surface and also examined the same for unsteady case. Hayat et al. [9] discussed the flow and developed the convergent solution of series for a Casson fluid in the presence of nanoparticles and radiation.

Hamid et al. [10] attained the dual solutions for the flow and transport of heat of Casson fluid. They have perceived that radiation is a factor affecting both steady and unsteady flow. Aneja et al. [11] made an analysis of natural convection in a porous cavity that was partially heated for Casson fluid flow. The coupled equations formed are solved by the implementation of penalty FEM method. Sreelakshmi et al. [12] investigated the Buongiorno model for free convection on Casson flow on a radiated-elastic surface. Nagaraja and Gireesha [13] studied the behaviour of such fluid over curved surface that can be stretched with the impact of heat production on MHD Casson fluid flow. The result attained by them is worth noticing for future advancement.

Suspending tiny solid particles to the base fluid assists in the energy transmission, fluids can improve their thermal conductivity and thus this provides an effective and innovative way to enhance the heat transfer characteristics significantly. Buongiorno model exemplifies the impact of nano dimensional particle due to the phenomenon of Brownian motion and thermophoretic diffusion. Buongiorno [14] studied that the base fluids thermal conductivity can be magnified in an unnatural way by embedding nanoparticles into them. Maghrebi et al. [15] came up with the forced convection heat transfer of nanofluids in a porous channel. Darcy-Brinkman-Forchheimer approach is implemented to study the porous behaviour. Schio et al. [16] made research on the consequences of Brownian and thermophoresis diffusion on the laminar forced convective flow in a channel. Their analysis revealed that if the boundary temperature is linearly varied the concentration no longer is the function of temperature for prescribed Peclet number value.

Nield and Kuznetsov [17] studied the forced convection in a parallel-plate channel assuming streamwise varying temperature boundary conditions engaged by a nanofluid. Swain et al. [18] used this model to report the consequence of allowing the Williamson nanofluid through porous medium consideration of irregular heat source/sink. Kho et al. [19] looked into transmission of heat and mass when Williamson nanofluid is passed over the stretched surface. Chu et al [20] gave away the importance of activation energy, bio-convection and third grade fluid flowing over a stretched surface. Their work has reported that thermal field is enlarged by augmentation in Brownian movement and thermophoretic diffusion. This model was also implemented by Javed and Farooq [21] for their study on melting rheology in two-fold stratified Eyring-Powell flow over surface when the thickness is varied.

Abbassi [22] analyzed entropy generated in a regularly heated microchannel heat sink (MCHS). As a criteria for the functioning of the system they have discussed second law analysis. Sciacovelli et al [23] carried out the study on entropy to decipher its importance in designing engineering tools. They have focussed on minimising entropy generated within the system. Bhatti et al. [24] presented a model theoretically to determine the entropy produced on electro-kinetically modulated peristaltic propulsion on the magnetized nanofluid flow through a microchannel with Joule heating. Manay et al. [25] investigated the effects when nano sized $\mathrm{TiO} 2$ parti- 
cles is immersed in the base fluid in a MCHS. Liu et al. [26] studied the entropy generation analysis of electro-magneto hydrodynamic (EMHD) flow of Newtonian fluids through a bent rectangle shaped microchannel. Samal and Moharana [27] made a numerical study on entropy produced in recharging as well as simple microchannel to explore effect of geometrical alteration on thermodynamic irreversibility in a system. Gireesha et al. [28] scrutinized entropy of couple stress liquid flowing in an upright microchannel.

The present work portrays the impacts of Casson nanofluid flowing along the horizontal microchannel whose walls promote simultaneous injection/suction of the fluid. Microchannel is incorporated with porous media by implementing Darcy-Forchheimer model. Consequences of two mechanisms namely Brownian motion and thermophoresis on the flowing fluid along with the transport of mass and heat is accounted. Convective and no slip boundary conditions are considered. The consequential outcomes are sketched in the graphs provided.

\section{Mathematical Formulation}

A Casson nanofluid flow between two horizontal microchannel comprised of two plates of infinite length placed parallelly separated by a distance $a$, as shown in the figure. Here the fluid flow through the microchannel is regarded as steady, laminar, viscous and incompressible. The fluid movement is due to the pressure force on the flow. Here, the nanofluid suction and injection occurs uniformly at the upper and lower plates of microchannel respectively. The lower plate exchanges heat by convection with warm fluid at temperature $T_{h}$ and the upper plate is kept at ambient temperature $T_{a}$ as it exchanges heat with the surrounding fluid. In order to study heat and mass transfer, Buongiorno model is incorporated. It emphasizes on Brownian motion and thermophoretic mechanisms occurring in the fluid. Figure 1 describes the flow.
For solving these equations, we have assumed that the velocity agrees slip condition at the plates and the heat transfer equation agrees with the convective conditions.

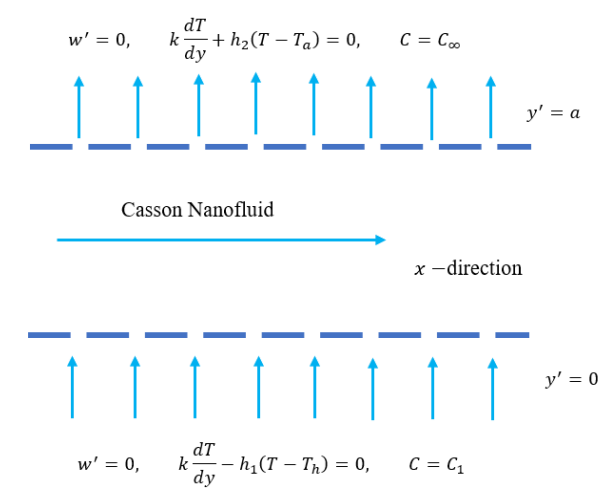

Figure 1: Physical Interpretation of the flow

The governing equations for above described incompressible Casson nanofluid flow are,

$$
\begin{gathered}
v_{0} \frac{d w^{\prime}}{d y^{\prime}}=-\frac{1}{\rho_{f}} \frac{d p}{d x}+\left(1+\frac{1}{\beta}\right) \frac{d^{2} w^{\prime}}{d y^{\prime 2}} \\
-\frac{v}{K} w^{\prime}-\frac{F^{*}}{\sqrt{K}} w^{\prime 2}
\end{gathered}
$$

$\nu_{0} \frac{d T}{d y^{\prime}}=\frac{k}{\left(\rho C_{p}\right)_{f}} \frac{d^{2} T}{d y^{\prime 2}}+\frac{\mu}{\left(\rho C_{p}\right)_{f}}\left(1+\frac{1}{\beta}\right)\left(\frac{d w^{\prime}}{d y^{\prime}}\right)^{2}$

$+\tau\left[D_{B} \frac{d T}{d y^{\prime}} \frac{d C}{d y^{\prime}}+\frac{D_{T}}{T_{a}}\left(\frac{d T}{d y^{\prime}}\right)^{2}\right]$

$v_{0} \frac{d C}{d y^{\prime}}=D_{B} \frac{d^{2} C}{d y^{\prime 2}}+\frac{D_{T}}{T_{a}} \frac{d^{2} T}{d y^{\prime 2}}$

The entropy production equation becomes,

$$
\begin{aligned}
& E_{G}=\frac{k}{T_{a}^{2}}\left(\frac{d T}{d y^{\prime}}\right)^{2}+\frac{\mu}{T_{a}}\left(1+\frac{1}{\beta}\right)\left(\frac{d w^{\prime}}{d y^{\prime}}\right)^{2} \\
& +\frac{R D_{B}}{C_{\infty}}\left(\frac{d C}{d y^{\prime}}\right)^{2}+\frac{R D_{B}}{T_{a}}\left(\frac{d T}{d y^{\prime}}\right)\left(\frac{d C}{d y^{\prime}}\right)
\end{aligned}
$$

At the channel extremes no-slip and convective boundary conditions are assumed, 


$$
\begin{array}{ll}
w^{\prime}(0)=0, k\left(\frac{d T}{d y^{\prime}}\right)-h_{1}\left(T-T_{h}\right)=0 & N s=\left(\frac{d \theta}{d y}\right)^{2}+E c \operatorname{Pr} \gamma\left(1+\frac{1}{\beta}\right)\left(\frac{d w}{d y}\right)^{2} \\
C=C_{1} \text { at } y^{\prime}=0, \quad(5) & \delta \omega \gamma\left(\frac{d \theta}{d y}\right)\left(\frac{d \phi}{d y}\right)+\delta \omega^{2} \gamma^{2}\left(\frac{d \phi}{d y}\right)^{2} \\
w^{\prime}(1)=0, k\left(\frac{d T}{d y^{\prime}}\right)+h_{2}\left(T-T_{a}\right)=0, & \text { with boundary conditions, }
\end{array}
$$$$
C=C_{\infty} \text { at } y^{\prime}=1
$$

where $w^{\prime}-$ axial velocity, $\vartheta_{0}$-suction/injection velocity, $\mu, \rho, C_{p}$ - dynamic viscosity, density, specific heat at constant pressure, $\beta$ - Casson parameter, $D_{T}$-thermophoresis co-efficient, $D_{B}$-Brownian movement co-efficient, $C_{1}$ and $C_{\infty}$ - concentrations at the wall and surrounding respectively, and $h_{1}$ and $h_{2}$ heat transfer co-efficient of the lower and upper plates likewise, $\quad F^{*}$-Forcheimer co-efficient, $K$-porous media permeability.

In order to make dimensions of the above equations to one, following variables are used.

$$
\begin{aligned}
& w=\frac{\rho_{f} a w^{\prime}}{\mu_{f}}, y=\frac{y^{\prime}}{a}, \theta=\frac{\left(T-T_{a}\right)}{\left(T_{h}-T_{a}\right)}, \\
& \phi=\frac{\left(C-C_{\infty}\right)}{\left(C_{1}-C_{\infty}\right)}
\end{aligned}
$$

with boundary conditions,

$$
\begin{aligned}
& w(0)=0, \frac{d \theta}{d y}-B i(\theta-1)=0, \phi(0)=1 \text { at } \\
& y=0,
\end{aligned}
$$

$w(1)=0, \frac{d \theta}{d y}+B i \theta=0, \phi(1)=0$ at $y=1(13)$

In above equations, $P=\frac{a^{3} \rho}{\mu^{2}}\left(-\frac{d p}{d x^{\prime}}\right)-$ pressure gradient parameter, $R e=\frac{v_{0} a \rho}{\mu}$ Reynolds number, $\operatorname{Pr}=\frac{\mu C_{p}}{k}-$ Prandtl number, $D a=\frac{K}{a^{2}}-$ Darcy number, $P e=R e P r-$ Peclet number, $\quad \gamma=\frac{T_{a}}{T_{h}-T_{a}}-$ temperature difference parameter, $E c=$ $\frac{\vartheta^{2}}{c_{p}\left(T_{h}-T_{a}\right) a^{2}}-$ Eckert number, $N b$ $=\frac{\tau D_{B}\left(C_{1}-C_{\infty}\right)}{\vartheta}$ Brownian motion parameter, $N t=\frac{\tau D_{T}\left(T_{h}-T_{a}\right)}{\vartheta T_{a}}-$ thermophoresis $\operatorname{Re}\left(\frac{d w}{d y}\right)=P+\left(1+\frac{1}{\beta}\right)\left(\frac{d^{2} w}{d y^{2}}\right) \quad$ parameter, $\quad S c=\frac{\vartheta}{D_{B}}-$ Schmidt $\quad$ number, $B i=\frac{a h_{i}}{k}$ - Biot number where $i=1,2$ for lower and upper plates correspondingly, $\delta=$ $\frac{R D_{B} C_{\infty}}{k}-$ diffusion parameter, $\omega=\frac{C_{1}-C_{\infty}}{C_{\infty}}-$ concentration difference ratio.

$$
\begin{aligned}
& \frac{d^{2} \theta}{d y^{2}}-\operatorname{RePr}\left(\frac{d \theta}{d y}\right)+E c \operatorname{Pr}\left(1+\frac{1}{\beta}\right)\left(\frac{d w}{d y}\right)^{2} \\
& +N b \operatorname{Pr}\left(\frac{d \theta}{d y}\right)\left(\frac{d \phi}{d y}\right)+N t \operatorname{Pr}\left(\frac{d \theta}{d y}\right)^{2}=0
\end{aligned}
$$

$$
\frac{d^{2} \phi}{d y^{2}}+\frac{N t}{N b} \frac{d^{2} \theta}{d y^{2}}-S c \operatorname{Re}\left(\frac{d \phi}{d y}\right)=0
$$

Since we have $N s=N_{h}+N_{f}+N_{m}$,

where $N_{h}=\left(\frac{d \theta}{d y}\right)^{2}$ irreversibility due to heat transfer, 


$$
N_{f}=E c \operatorname{Pr} \gamma\left(1+\frac{1}{\beta}\right)\left(\frac{d w}{d y}\right)^{2}
$$

irreversibility due to fluid friction,

$$
N_{m}=\delta \omega \gamma\left(\frac{d \theta}{d y}\right)\left(\frac{d \phi}{d y}\right)+\delta \omega^{2} \gamma^{2}\left(\frac{d \phi}{d y}\right)^{2}
$$

irreversibility due to mass.

Thus, Bejan number,

$$
B e=\frac{N_{h}}{N_{h}+N_{f}+N_{m}}
$$

\section{Numerical Procedure}

One way to promise the solution of IVP to be precise is to solve the problem twice using step size $h$ and $h / 2$. But this process requires substantial simulation for the smaller step size and is carried out until the result is in good agreement. This numerical scheme i.e., RungeKutta Fehlberg scheme is one such method which has a procedure to decide if the correct step size is used. At every step, 2 distinct approximations are obtained for the solution and compared. If the two responses are in close agreement, then approximation obtained is accepted if not, step size is lessened. The step size is magnified if the answer settles to more significant digits. Below 6 values are achieved

in each step: $k_{1}=h f\left(x_{i}, y_{i}\right)$,

$k_{2}=h f\left(x_{i}+\frac{h}{24}, y_{i}+\frac{\mathrm{k}_{1}}{4}\right)$,

$k_{3}=h f\left(x_{i}+\frac{3 h}{8}, y_{i}+\frac{3 \mathrm{k}_{1}}{32}+\frac{9 k_{2}}{32}\right)$,

$k_{4}=h f\left(x_{i}+\frac{12 h}{13}, y_{i}+\frac{1932 \mathrm{k}_{1}}{2197}-\frac{7200 k_{2}}{2197}+\frac{7296 k_{3}}{2197}\right)$,

$k_{5}=h f\left(x_{i}+h, y_{i}+\frac{439 \mathrm{k}_{1}}{216}-8 k_{2}+\frac{3680 k_{3}}{513}-\frac{845 k_{4}}{4104}\right)$,

$k_{6}=h f\left(x_{i}+\frac{h}{2}, y_{i}+\frac{8 \mathrm{k}_{1}}{27}+2 k_{2}-\frac{3544 k_{3}}{2565}-\frac{1859 k_{4}}{4104}-\frac{11 k_{5}}{40}\right)$,

Then an approximation using $4^{\text {th }}$ order RKmethod is,

$$
y_{i+1}=y_{i}+\frac{25 k_{1}}{216}+\frac{1408 k_{3}}{2565}+\frac{2197 k_{4}}{4104}-\frac{k_{5}}{5},
$$

It is noteworthy that $k_{2}$ value is not counted in the above given formula. The other value of $y$ is known by $5^{\text {th }}$ order RK-method as:

$$
y_{i+1}^{*}=y_{i}+\frac{16 k_{1}}{135}+\frac{6656 k_{3}}{12825}+\frac{28561 k_{4}}{56430}-\frac{9 k_{5}}{50}+\frac{2 k_{6}}{55},
$$

If $\left|y_{i+1}+y_{i+1}^{*}\right|$ is small enough, then the method is terminated; or else the simulation is carried on using lesser step size $h$. The local truncation error is $y_{i+1}-y_{(i+1)}^{*}$.

\section{Result and Discussion}

Flow of Casson fluid in the horizontal microchannel with influence of Brownian movement and thermophoresis when the channel is incorporated with Darcy Forchheimer model is elaborated. The equations that we get are solved by making use of RKF-method in association with shooting procedure. The outcomes of the flow are discussed in this section.

Velocity profile exhibits parabolic nature. At the extremes of the microchannel the flow velocity deteriorates with the excess but at the centre there is magnification in the flow velocity. Behaviour of $R e$ Reynolds number on flow velocity is represented in figure 2 . There is decrement in the velocity at the fluid injecting wall and increment in velocity at fluid sucking wall of the channel. Consequence of rising Casson parameter on velocity of the fluid can be seen in figure 3. Higher the Casson parameter the more is the possibility of the fluid to behave as Newtonian fluid as the term $1 / \beta$ tends to zero. Thus, higher the Casson parameter value more is the velocity of the fluid. Figure 4 describes that on rising Darcy number, flow is facilitated well. This is because, permeability of porous medium enhances, leading to the cut down in friction with this medium. 


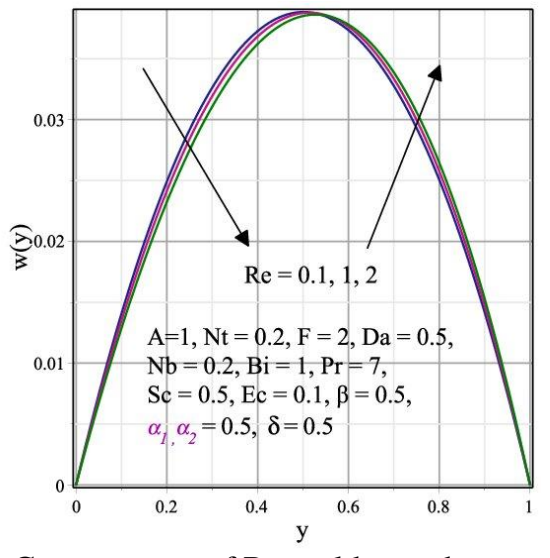

Figure 2: Consequence of Reynolds number on velocity profile $\boldsymbol{w}(\boldsymbol{y})$

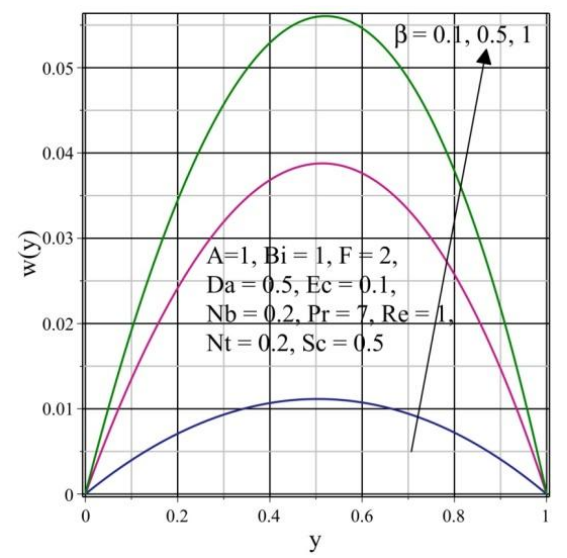

Figure 3: Consequence of Casson parameter on velocity profile $\boldsymbol{w}(\boldsymbol{y})$

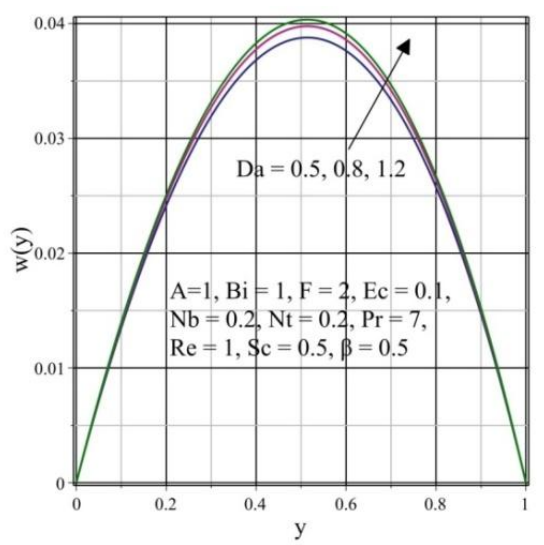

Figure 4: Consequence of Darcy number on velocity profile $\boldsymbol{w}(\boldsymbol{y})$

Figure 5 shows that the velocity decreases with the increase in the value of Forchheimer co-efficient $F^{*}$ and is maximum at the centre of the channel. Onenhancing $F^{*}$ we observe depletion in ow velocity since the augmentation of the inertial effects rises the drag of the porous media which results in magnifying the resistance to the flow.

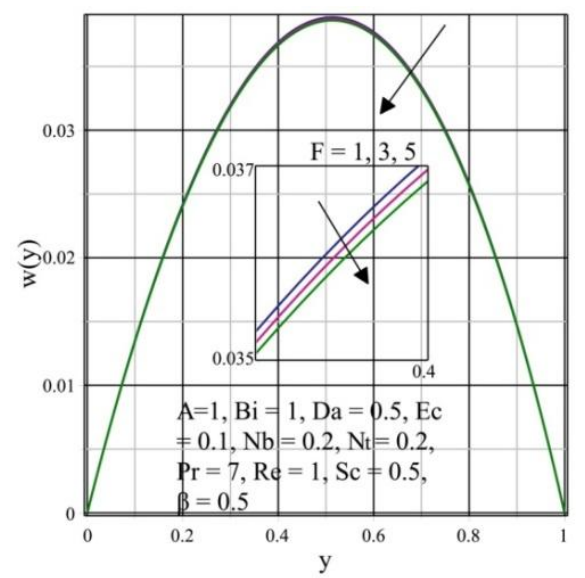

Figure 5: Consequence of Forchheimer co-efficient on velocity profile $\mathrm{w}(\mathrm{y})$

Entropy generated is high at the suction wall and low at injection wall. The profile shows not much deviation at the centre of the microchannel for varying parametric values, but significant deviations can be observed at the walls. Bejan number profile is maximum at the centre of the microchannel and minimum at the boundaries which signifies the dominance of heat transfer and mass transfer irreversibility at the centre of the microchannel.

Figure 6(a) gives the effect of Casson parameter $\beta$ on thermal profile. On magnifying temperature elevates.

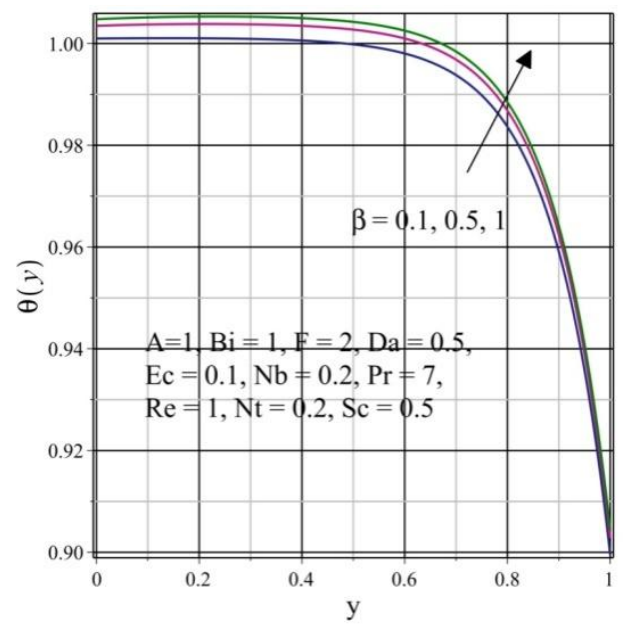

Figure 6(a): Consequence of Casson parameter on temperature profile $\boldsymbol{\theta}(\boldsymbol{y})$ 
Figures 6(b) and 6(c) depict the profile of entropy generation and Bejan number for varying $\beta$. Maximum entropy is produced when $\beta$ value is high. As the Casson parameter increases, the Bejan number decreases which states that for higher value of Casson parameter maximum rreversibility is contributed due to fluid friction.

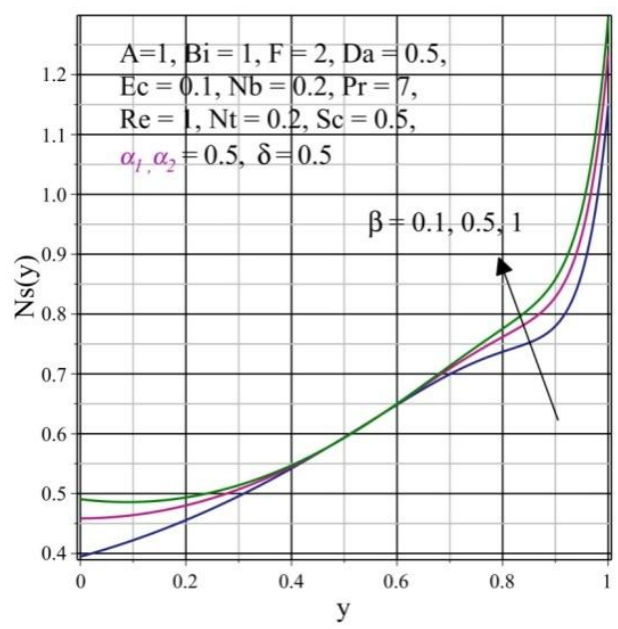

Figure 6(b): Consequence of Casson parameter on entropy generation profile $\boldsymbol{N} \boldsymbol{s}(\boldsymbol{y})$

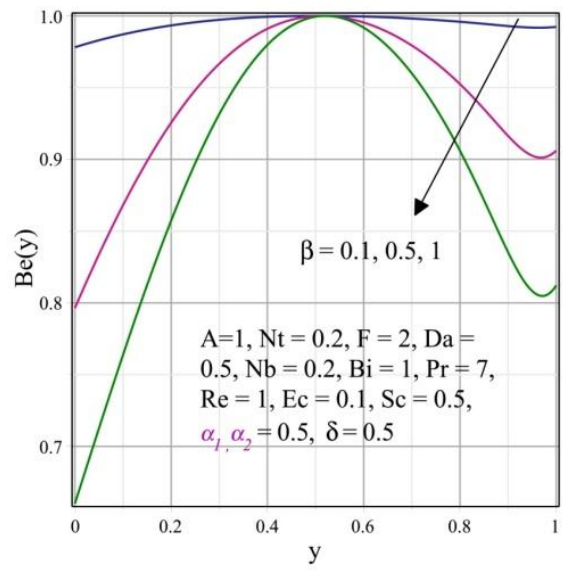

Figure 6(c): Consequence of Casson parameter on Bejan number profile $\boldsymbol{B} \boldsymbol{e}(\boldsymbol{y})$

Profiles of temperature, entropy rate and Bejan number is on display in the figures $7(a)$, (b) and (c) respectively. By the augmentation of $\mathrm{Bi}$, depletion in temperature is attained. The reason behind this is transport of heat occurring from warm nano-dimensional particles to relatively cold nano-dimensional particles. This can be ascribed to the cooling effects by the convective heat loss. Also, for increasing $B i$, entropy enhances which is evident from the reason that higher value of $B i$ promotes convection. From figure 7(c) Bejan number profile maximizes for higher $B i$ value. For high $B i$ value irreversibility due to heat transfer is more.

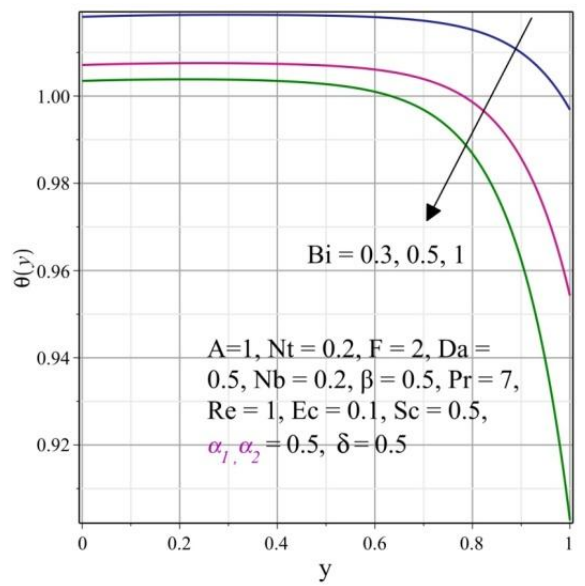

Figure 7(a): Consequence of Biot number on temperature profile $\boldsymbol{\theta}(\boldsymbol{y})$

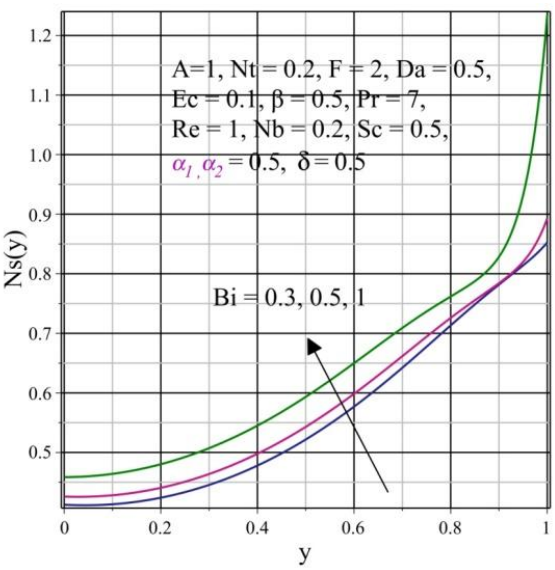

Figure 7(b): Consequence of Biot number on entropy generation profile $\boldsymbol{N} \boldsymbol{s}(\boldsymbol{y})$

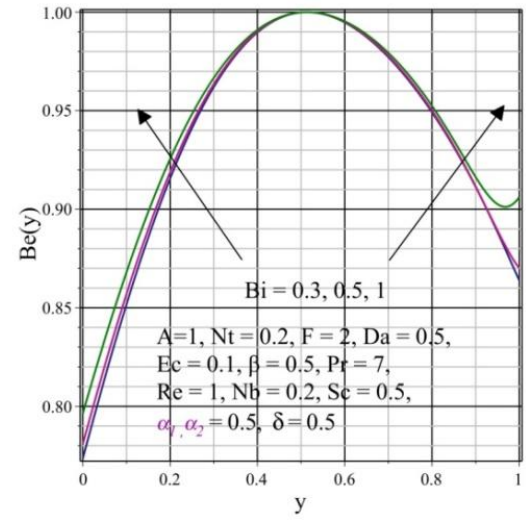

Figure 7(c): Consequence of Biot number Bejan number profile $\boldsymbol{B} \boldsymbol{e}(\boldsymbol{y})$

Figures 8(a), (b) and (c) are representation of 
varying $E c$ values on thermal, entropy and Bejan number profile. Elevation in thermal and entropy profile is perceived for higher values of $E c$. This is the result of viscous dissipation effect. Higher the $E c$ value more is viscous heating causing rise in temperature and as a result rise in entropy. Ratio of viscous force to inertial force gives Eckert number. When the viscous force of the fluid is utmost randomness of the particles is more which causes the loss of thermal energy. Higher the viscous dissipation larger is the irreversibility caused in the microchannel and irreversibility due to fluid friction is dominant throughout the microchannel.

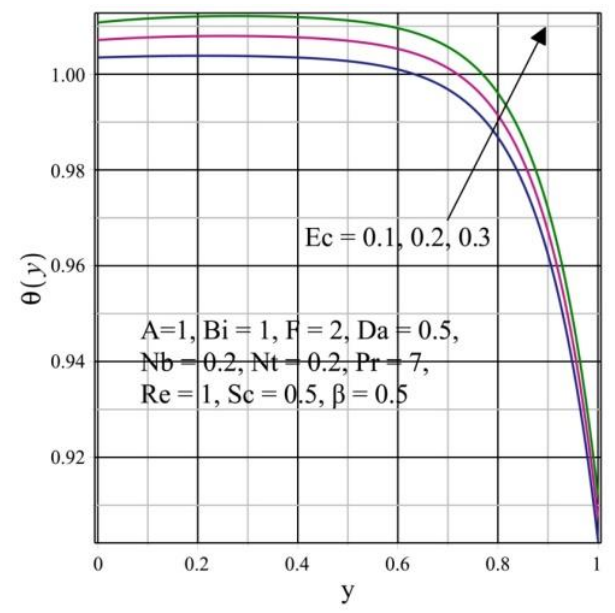

Figure 8(a): Consequence of Eckert number on temperature profile $\boldsymbol{\theta}(\boldsymbol{y})$

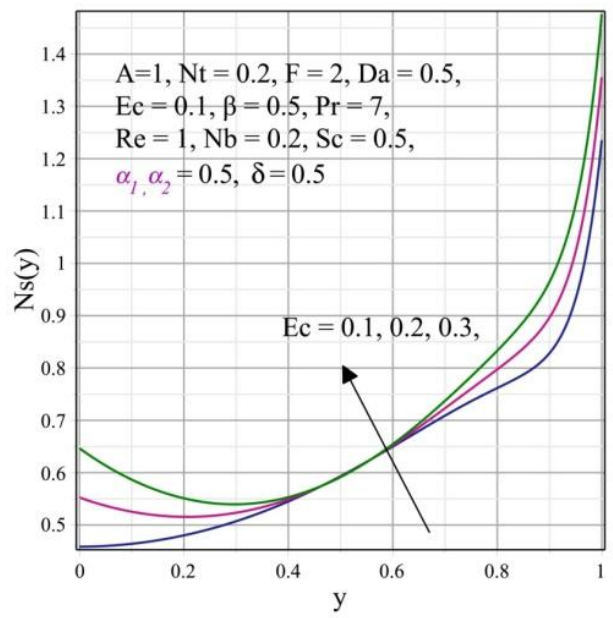

Figure 8(b): Consequence of Eckert number on entropy generation profile $\boldsymbol{N} \boldsymbol{s}(\boldsymbol{y})$

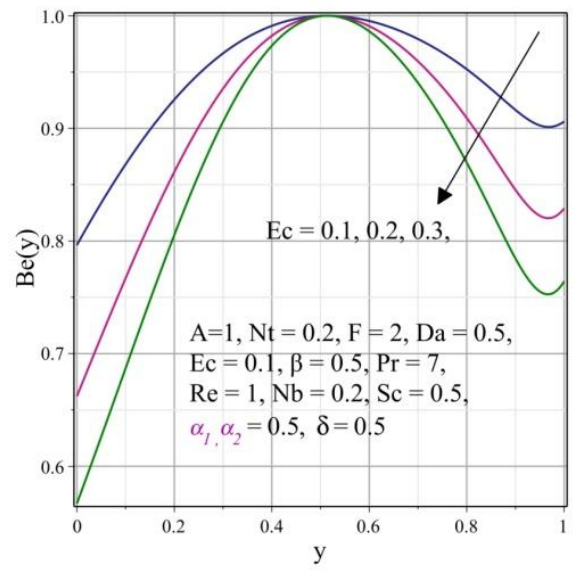

Figure 8(c): Consequence of Eckert number on Bejan number profile $\boldsymbol{B} \boldsymbol{e}(\boldsymbol{y})$

Figures 9 (a) and (b) illustrates the temperature and concentration to maximize with the rise $\mathrm{Nb}$. As $\mathrm{Nb}$ magnifies, less heat is transferred to the walls, thus there is elevation in temperature as there is random gesture of fluid particles. Increment of concentration with larger values of $\mathrm{Nb}$ is because of therandom motion of nano-sized particles which prohibit them to settle down, thus mass flux rises, consequently concentration is observed to rise throughout the flow. Entropy generated is low at the lower plate and high at the upper plate for increasing $N b$ as shown in figure 9 (c). Similar behavior is retained for Bejan number profile in figure 9 (d) as well. Thus, for large values of $\mathrm{Nb}$ irreversibility caused due to fluid friction is more at the lower wall and least at the top wall.

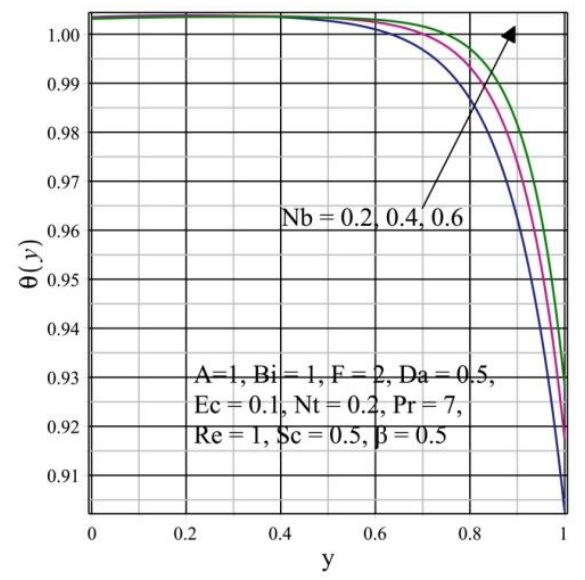

Figure 9(a): Consequence of Brownian motion parameter on temperature profile $\theta(y)$ 


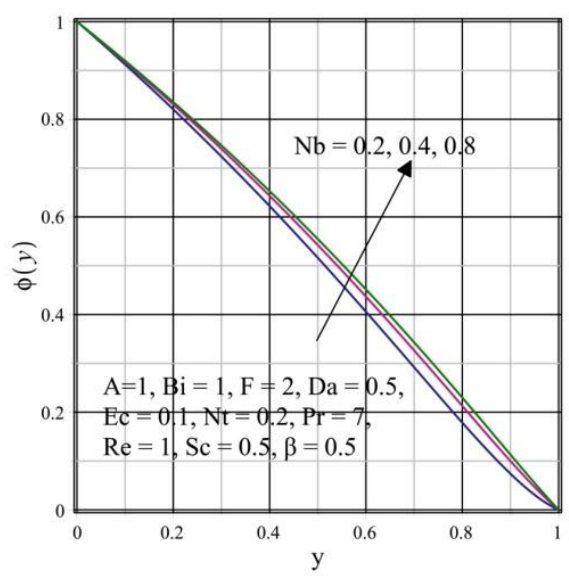

Figure 9(b): Consequence of Brownian motion parameter on concentration profile $\phi(y)$

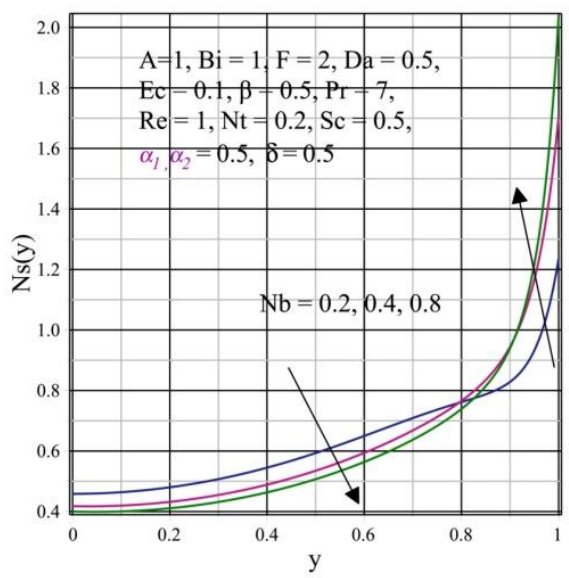

Figure 9 (c): Consequence of Brownian motion parameter on entropy generation profile $N s(y)$

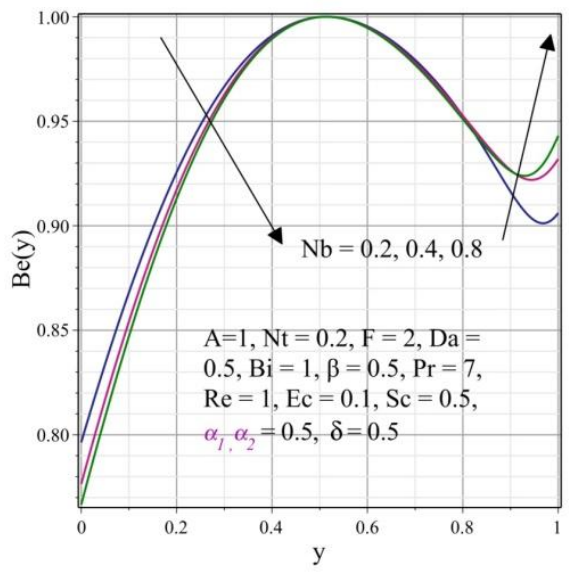

Figure 9(d): Consequence of Brownian motion parameter on Bejan number profile $B e(y)$

Thermal profile shows enhancing nature for higher $N t$ value which is disclosed in figure 10 (a) but the reverse behavior is attained for concentration profile. Thermophoresis is a mechanism which arrives when there is existence of thermal gradient. This process involves deposition of particles onto the cold surfaces. During the process a concentration gradient originates and the profile for concentration is noticed to be increasing one as in figure $10(b)$.

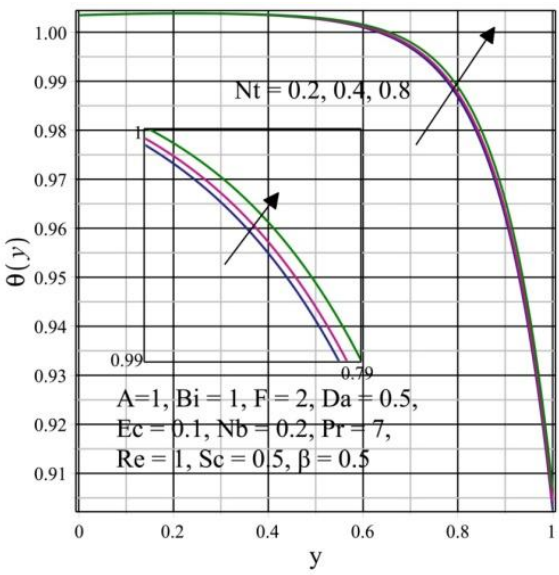

Figure 10(a): Consequence of thermophoresis parameter on temperature profile $\theta(y)$

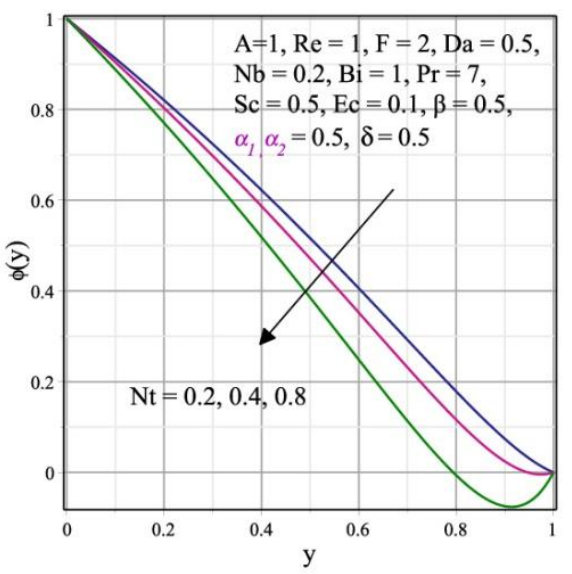

Figure 10(b): Consequence of thermophoresis parameter on concentration profile $\phi(y)$

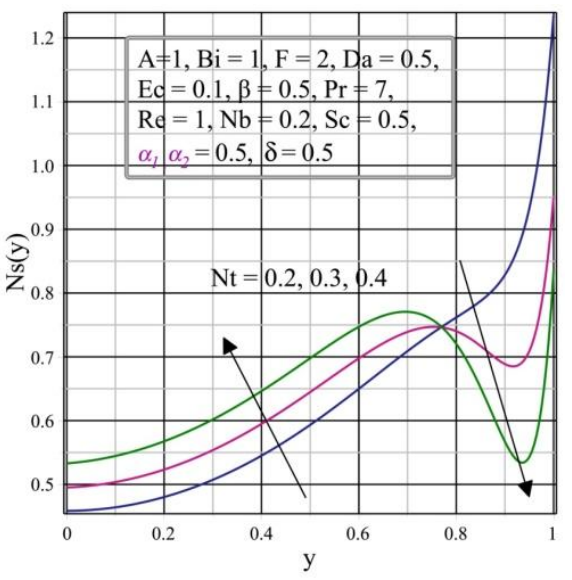

Figure 10 (c): Consequence of thermophoresis parameter on entropy generation profile $N s(y)$ 
The entropy is high at the bottom walls and low at the top walls as there is diffusion of the particles to the cold surface i.e., at the suction wall, the profile exhibits decreasing to increasing nature all through the microchannel as in figure 10 (c). Similar behavior is noticed in figure $10(\mathrm{~d})$ for $\mathrm{Be}$ profile.

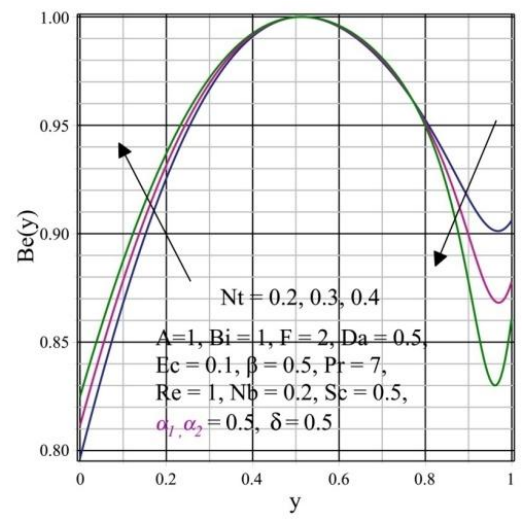

Figure 10(d): Consequence of thermophoresis parameter on Bejan number profile $B e(y)$

Figure 11(a) elucidates the conduct of Schmidt number $S c$ on concentration profile. It is as expected because Schmidt number is the ratio of momentum diffusivity to mass diffusivity which implies that rise in $S c$ is nothingbut rise in momentum diffusivity.

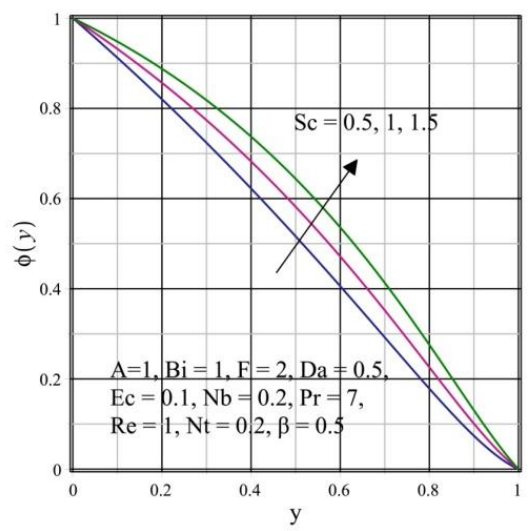

Figure 11(a): Consequence of Schmidt number on concentration profile $\phi(y)$

Apparently, for higher value of Schmidt number irreversibility is low on lower side of the channel whereas for same Schmidt number irreversibility is high at upper side of the channel. That is Sc is depleting function on bottom and magnifying function at the top for both entropy generation and Bejan number as in figures 11(b) and 11(c).

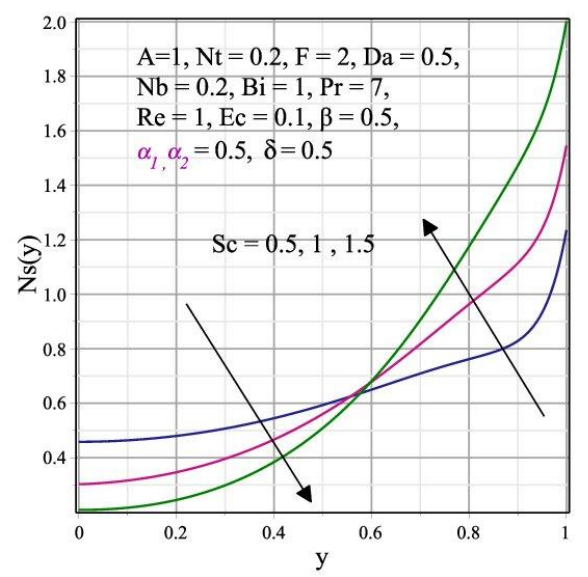

Figure 11(b): Consequence of Schmidt number entropy generation profile $N s(y)$

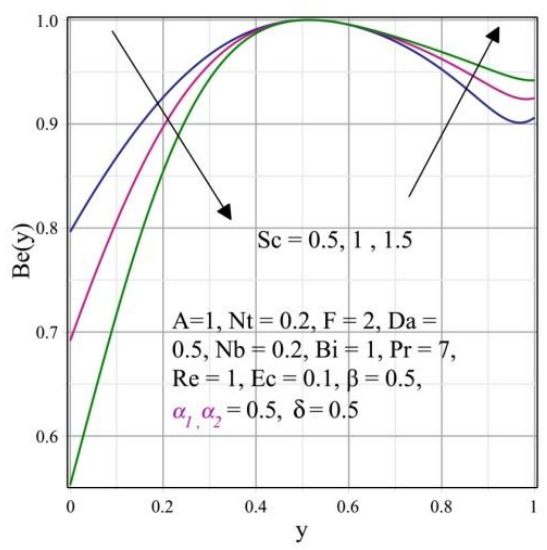

Figure 11(c): Consequence of Schmidt number on Bejan number profile $B e(y)$

\section{Concluding Reviews}

The study discloses the flow of Casson nanomaterial flowing through the horizontal microchannel with the effect of Brownian movement and thermophoresis when subjected to no slip and convective conditions at the extremes of the microchannel. Darcy Forchheimer model is implemented. Thus, major results can be listed as:

- The velocity of the non-Newtonian fluid considered is noticed to elevate with $D a$ and deplete with Forchheimer co-efficient $F$.

- The Casson parameter $\beta$ increases the temperature and entropy all through the microchannel, whereas it decreases the Bejan 
number. For the higher value of it, the fluid starts to behave as Newtonian fluid.

- Thermal profile is an elevating function for $E c, N b$ and $N t$ and depleting functionof $B i$.

- $S c$ and $N b$ maximizes the profile of concentration but $N t$ minimizes the same.

- The Biot number $B i$ increases the entropy and Bejan number profile.

Bejan number diagrams depict the plots to deplete at the bottom wall and elevate at the top wall for varying $N b$ and $S c$. Contrary nature is gained in case of $N t$.

\section{References}

1. P. Forchheimer, Wasserbewegung durch boden. Z. Ver. Deutsch, Ing., Vol. 45, 1901 pp. 1782-1788.

2. V. Girault, M. F. Wheeler, Numerical Discretization of a Darcy-Forchheimer Model, Numerische Mathematik, Vol. 110, No. 2, 2008 pp. 161-198.

3. P. Knabner, J. E. Roberts, Mathematical Analysis of a Discrete Fracture Model Coupling Darcy Flow in the Matrix with Darcy-Forchheimer Flow in the Fracture, ESAIM: Mathematical Modelling and Numerical Analysis, Vol. 48, No. 5, 2014 pp. 1451-1472.

4. R. S. Saif, T. Hayat, R. Ellahi, T. Muhammad, A. Alsaedi, Darcy-Forchheimer Flow of Nanofluid due to a Curved Stretching Surface, International Journal of Numerical Methods for Heat \& Fluid Flow, Vol. 29 No. 1, 2019, pp. 2-20.

5. M. K. Nayak, F. Mabood, I. Tlili, A. S. Dogonchi, W. A. Khan, Entropy Optimization Analysis on Nonlinear Thermal Radiative Electromagnetic Darcy-Forchheimer Flow of SWCNT/MWCNT Nanomaterials, Applied Nanoscience, Vol. 11, No. 2, 2021, pp.399-418.
6. N. Casson, A Flow Equation for Pigment Oil Suspensions of the Printing Ink Type, Rheology of Disperse Systems, Pergamon press, New York, 1959, pp.84-104.

7. M. Mustafa, T. Hayat, I. Pop, S. Asghar, S. Obaidat, Stagnation-Point Flow of a Nanofluid towards a Stretching Sheet, International Journal of Heat and Mass Transfer, Vol. 54, No. (25-26), 2011, pp. 5588-5594.

8. S. Mukhopadhyay, Casson Fluid Flow and Heat Transfer over a Nonlinearly Stretching Surface, Chinese Physics B, Vol. 22 No. 7 2013074701

9. T. Hayat, S. Asad and A. Alsaedi, Flow of Casson Fluid with Nanoparticles, Applied Mathematics and Mechanics, Vol. 37, No. 4, 2016, pp. 459-470.

10. M. Hamid, M. Usman, Z. H. Khan, R. Ahmad, W. Wang, Dual Solutions and Stability Analysis of Flow and Heat Transfer of Casson fluid over a Stretching Sheet, Physics Letters A, Vol. 383, No. 20, 2019, pp. 2400-2408.

11. M. Aneja, A. Chandra, S. Sharma, Natural Convection in a Partially Heated Porous Cavity to Casson fluid, International Communications in Heat and Mass Transfer, Vol. 114, 2020.pp.1-15.

12. T. K. Sreelakshmi, A. Abraham,C. S. K. Raju, B. C. Prasannakumara, A. S. Chethan, Brownian Motion and Thermophoresis Significance of Casson Fluid Flow in an Elastic-Radiated Surface with a Suspension of Transpiration and Non-Fourier Heat Flux, Waves in Random and Complex Media, 2021, pp. 1-20.

13. B. Nagaraja, B. J. Gireesha, Exponential Space-dependent Heat Generation Impact on MHD Convective Flow of Casson Fluid over a Curved Stretching Sheet with Chemical Reaction, Journal of Thermal Analysis \& Calorimetry, Vol.143, No.6, 2021, pp. 40714079 
14. J. Buongiorno, Convective Transport in Nanofluids, Journal of Heat Transfer, Vol. 128, 2006, pp. 240-250.

15. M. J. Maghrebi, M. Nazari, T. Armaghani, Forced Convection Heat Transfer of Nanofluids in a Porous Channel, Transport in Porous Media, Vol. 93, No.3, 2012, pp. 401413.

16. E. Rossi di Schio, M. Celli, A. Barletta, Effects of Brownian Diffusion and Thermophoresis on the Laminar Forced Convection of a Nanofluid in a Channel, Journal of Heat Transfer, Vol. 136, No. 2, 2013, pp. 022401-022410

17. D. A. Nield, A. V. Kuznetsov, Forced Convection in a Parallel-Plate Channel occupied by a Nanofluid or a Porous Medium Saturated by a Nanofluid, International Journal of Heat and Mass Transfer, Vol. 70, 2014, pp. 430-433.

18. K. Swain, S. K. Parida, G. C. Dash, Effects of Non-Uniform Heat Source/Sink and Viscous Dissipation on MHD Boundary Layer Flow of Williamson Nanofluid through Porous Medium, Defect and Diffusion Forum, Vol. 389, 2018, pp.110-127,

19. Y. B. Kho, A. Hussanan, M. K. A. Mohamed, M. Z. Salleh, Heat and Mass Transfer Analysis on Flow of Williamson Nanofluid with Thermal and Velocity Slips: Buongiorno Model, Propulsion and Power Research, Vol. 8, No. 3, 2019, pp.243-252.

20. Y. M. Chu, M. I. Khan, N. B. Khan, S. Kadry, S. U. Khan, I. Tlili, M. K. Nayak, Significance of Activation Energy, Bio-convection and Magnetohydrodynamic in Flow of Third Grade Fluid (non-Newtonian) towards Stretched Surface: A Buongiorno Model Analysis, International Communications in Heat and Mass Transfer, Vol. 118, 2020, pp.104893.
21. M. Javed, M. Farooq, Mixed Convection and Melting Rheology in Dual Stratified Eyring-Powell Nanofluid Flow over Surface of Variable Thickness: Buongiorno Model Approach, International Communications in Heat and Mass Transfer, Vol. 125, 2021, pp. 105322.

22. H. Abbassi, Entropy Generation Analysis in a Uniformly Heated Microchannel Heat Sink, Energy Journal, Vol. 32, No.10, 2007, pp.1932-1947.

23. A. Sciacovelli, V. Verda, E. Sciubba, Entropy Generation Analysis as a Design Tool-A Review, Renewable and Sustainable Energy Reviews, Vol. 43, 2015,pp. 1167-1181.

24. M. M. Bhatti, M. Sheikholeslami and A. Zeeshan, Entropy Analysis on Electro-Kinetically Modulated Peristaltic Propulsion of Magnetized Nanofluid Flow through a Microchannel, Entropy, Vol. 19, No. 9, 2017, 481

25. E. Manay, E. F. Akyurek, B. Sahin, Entropy Generation of Nanofluid Flow in a Microchannel Heat Sink, Results in Physics, Vol. 9, 2018, pp.615-624.

26. Y. Liu, Y. Jian, W. Tan, Entropy Generation of Electromagnetohydrodynamic (EMHD) Flow in a Curved Rectangular Microchannel, International Journal of Heat and Mass Transfer, Vol. 127, 2018, pp. 901-913.

27. S. K. Samal, M. K. Moharana, Second Law Analysis of Recharging Microchannel using Entropy Generation Minimization Method, International Journal of Mechanical Sciences, Vol.193, No.1, 2021, pp. 106174.

28. B. J. Gireesha, P. Venkatesh, F. Almeida, Entropy Scrutiny of Couple Stress Nanoliquid Flow with Slip and Convective Conditions in an Upright Microchannel, Physica Scripta, Vol. 96, No. 4, 2021, pp. 045302. 